\title{
Variable Speed Limits Control in an Urban Road Network to Reduce Environmental Impact of Traffic
}

\author{
Bassel Othman ${ }^{1,2}$, Giovanni De Nunzio ${ }^{1}$, Domenico Di Domenico ${ }^{1}$, Carlos Canudas-de-Wit ${ }^{2}$
}

\begin{abstract}
The problem of improving traffic sustainability and traffic efficiency in an urban road network, by implementing variable speed limits (VSL), is addressed in this paper. A nonlinear model predictive control (NMPC) design based on a first-order macroscopic traffic flow model is proposed for the speed limits optimization in each segment of the road network. Simulation results show the effectiveness of the proposed control approach, compared to reference cases in which the speed limits are constantly set to $30 \mathrm{~km} / \mathrm{h}$ or $50 \mathrm{~km} / \mathrm{h}$. In the particular case of congested traffic conditions, the controller is capable of reducing both energy consumption and travel time, without delaying users waiting at the network boundaries.
\end{abstract}

\section{INTRODUCTION}

Traffic congestion in large urban areas is one of the major problems cities are currently facing. Not only are road users spending more and more time in traffic jams, but congestion also poses health and environmental issues. Hence, more efficient ways of using road networks are under consideration to try and alleviate congestion and air pollution, such as speed limits control, traffic lights control, dynamic routing, and connected and automated vehicles [1].

It is well known that speed limits have an impact on traffic fluidity, energy consumption and pollutant emissions [2]. Variable speed limits (VSL) systems can be designed for either urban [3], [4] or highway [5], [6], [7] environments. Some authors have used VSL for ecological purposes [7], [4], but VSL strategies usually largely ignore this aspect, and rather focus on resolving traffic breakdown, and improving safety and throughput [8]. To the best of our knowledge, large-scale VSL based on a macroscopic traffic flow model and explicitly considering the environmental aspect in an urban scenario still represents a gap in the literature.

It is essential to bear in mind that a macroscopic traffic model does not capture all disturbances [1], [8], but it is still preferred to a microscopic model for complexity reasons. Macroscopic traffic flow models have been largely developed and validated in the literature [9]. For control applications, one of the moot points corresponds to the choice of the spatial discretization, especially for urban networks.

This study proposes a VSL control framework aimed at reducing the environmental impact and improving traffic fluidity. Simulations are performed in a synthetic urban network with signalized intersections and regulated turning

\footnotetext{
1 IFP Energies nouvelles, Rond-point de l'échangeur de Solaize, BP 3, 69360 Solaize, France. E-mail: \{bassel.othman, giovanni.de-nunzio, domenico.didomenico\}eifpen.fr

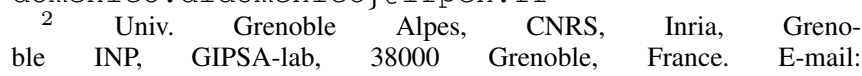
carlos.canudas-de-witegipsa-lab.fr
}

movements, which is a simplified approach, but which nevertheless offers a realistic complexity regarding movements at intersections. The performance of the controller is evaluated by comparing the resulting traffic situation with the constant $30 \mathrm{~km} / \mathrm{h}$ and $50 \mathrm{~km} / \mathrm{h}$ speed limits scenarios. Several methods can be used to implement VSL (reinforcement learning [5], receding horizon optimization [3], etc.), we consider a nonlinear model predictive control (NMPC) strategy that optimizes speed limits every 5 minutes. Traffic is depicted by a first-order macroscopic traffic flow model, namely the cell transmission model (CTM), based on the triangular fundamental diagram (FD) [10].

As in [11], the global energy consumption and NOx emissions computation has been adapted to account for accelerations within (temporal acceleration) and between (spatial acceleration) the cells of the traffic model. A feature of the proposed approach is that it not only improves the traffic performance metrics within the road network, but it also dedicates a special attention to the time spent by vehicles queuing at the network boundaries.

The body of the paper is organized as follows. Section II proposes a macroscopic first-order traffic model adapted for an urban environment. The ecological control design is presented in Section III, while Section IV provides and assesses experiments results. Finally, Section V contains concluding remarks and discussion on future perspectives.

\section{TRAFFIC MODEL}

\section{A. Network-wide cell transmission model}

To model road traffic, we consider the CTM [10], which is a first order Godunov-type [12] discretization of the LWR model. The model is extended to urban networks by considering traffic light signals (TLS) and the First In, First Out (FIFO) policy at intersections, as in [13]. The variables used to describe the problem are listed in Table I.

We consider an urban network of $n$ roads, each road being divided into $n_{\text {cells }}$ cells. There are three types of roads: inner roads of the network, roads entering the network, and roads exiting the network. Similarly, there are three types of cells: inner cells of a road, cells entering a road, and cells exiting a road. The following sets of cells are defined:

- $\mathcal{R}_{\text {enter }}^{\text {inner }}, \mathcal{R}_{\text {enter }}^{\text {in }}, \mathcal{R}_{\text {enter }}^{\text {out }}$ are the sets of cells entering respectively an inner, an entering, and an exiting road;

- $\mathcal{R}_{\text {exit }}^{\text {inner }}, \mathcal{R}_{\text {exit }}^{\text {in }}, \mathcal{R}_{\text {exit }}^{\text {out }}$ are the sets of cells exiting respectively an inner, an entering, and an exiting road;

- $\mathcal{R}$ is the set of all the cells of the urban network.

The operation of TLS at intersections is characterized by a function of time $\alpha(k)$, that regulates the outflow of the cells 
TABLE I: List of variables and parameters.

\begin{tabular}{|c|c|}
\hline Symbol & Description \\
\hline$D_{i}(k)$ & Demand of cell $i$ [veh s$\left.{ }^{-1}\right]$ \\
\hline$Q_{i}(k)$ & Number of vehicles queuing to enter cell $i$ [veh] \\
\hline$S_{i}(k)$ & Supply of cell $i$ [veh s ${ }^{-1}$ ] \\
\hline$T$ & Duration of simulation [time steps] \\
\hline$a_{\max }$ & Acceleration upper bound $\left[\mathrm{m} \mathrm{s}^{-2}\right]$ \\
\hline$a_{\min }$ & Acceleration lower bound $\left[\mathrm{m} \mathrm{s}^{-2}\right]$ \\
\hline$d_{i}(k)$ & Upstream demand on network entering cell $i\left[\mathrm{veh} \mathrm{s}^{-1}\right.$ ] \\
\hline$i$ & Cell index $[-]$ \\
\hline$k$ & Discrete time index $[-]$ \\
\hline$n$ & Number of roads in the network $[-]$ \\
\hline$n_{\text {cells }}$ & Number of cells in each road $[-]$ \\
\hline$s_{i}(k)$ & Downstream supply of exiting cell $i$ [veh s${ }^{-1}$ ] \\
\hline$u_{i}(k)$ & Speed limit in cell $i\left[\mathrm{~m} \mathrm{~s}^{-1}\right]$ [control] \\
\hline$u_{\max }$ & Speed limit upper bound $\left[\mathrm{m} \mathrm{s}^{-1}\right]$ \\
\hline$u_{\min }$ & Speed limit lower bound $\left[\mathrm{m} \mathrm{s}^{-1}\right]$ \\
\hline$u_{\text {ref }}$ & Speed limit of the baseline scenario $\left[\mathrm{m} \mathrm{s}^{-1}\right]$ \\
\hline$v_{i}(k)$ & Actual speed in cell $i\left[\mathrm{~m} \mathrm{~s}^{-1}\right]$ \\
\hline$w$ & Backward wave speed $\left[\mathrm{m} \mathrm{s}^{-1}\right]$ \\
\hline $\mathcal{N}_{i}$ & Set containing the downstream cells connected to $i[-]$ \\
\hline $\mathcal{P}_{i}$ & Set containing the upstream cells connected to $i[-]$ \\
\hline$\alpha_{i}(k)$ & Operation of TLS at the end of cell $i[-]$ \\
\hline$\beta_{i}$ & Split ratio of cell $i[-]$ \\
\hline$\delta_{t}$ & Discrete-time step size $[\mathrm{s}]$ \\
\hline$\delta_{x}$ & Cell length $[\mathrm{m}]$ \\
\hline$\rho_{i}(k)$ & Vehicle density in cell $i$ [veh $\mathrm{m}^{-1}$ ] \\
\hline$\rho^{\mathrm{cr}}$ & Critical density $\left[\mathrm{veh} \mathrm{m}^{-1}\right]$ \\
\hline$\rho^{\mathrm{M}}$ & Maximum vehicle density [veh $\mathrm{m}^{-1}$ ] \\
\hline$\varphi_{i}^{\text {in }}(k)$ & Traffic flow entering cell $i$ [veh s${ }^{-1}$ ] \\
\hline$\varphi_{i}^{\text {out }}(k)$ & Traffic flow exiting cell $i$ [veh s${ }^{-1}$ ] \\
\hline$\varphi^{\mathrm{M}}$ & Maximum traffic flow $\left[\mathrm{veh} \mathrm{s}{ }^{-1}\right.$ ] \\
\hline
\end{tabular}

in $\mathcal{R}_{\text {exit }}^{i n} \cup \mathcal{R}_{\text {exit }}^{\text {inner }}$, i.e. the cells exiting roads preceding an intersection. Hence, $\alpha$ can be expressed as

$$
\alpha:[0 . . T] \rightarrow\{0,1\}^{\left|\mathcal{R}_{\text {exit }}^{\text {in }} \cup \mathcal{R}_{\text {exit }}^{\text {inner }}\right|}
$$

where the output of the function at each time step is a vector of $\left|\mathcal{R}_{\text {exit }}^{\text {in }} \cup \mathcal{R}_{\text {exit }}^{\text {inner }}\right|$ values giving red or green (respectively 0 or 1). Function $\alpha$ is fully determined in advance, and must ensure the right of way at each time step

$$
\forall i \in \mathcal{R}_{\text {enter }}^{\text {inner }} \cup \mathcal{R}_{\text {enter }}^{\text {out }}, \sum_{r \in \mathcal{P}_{i}} \alpha_{r}(k)=1
$$

Note that we define $\alpha$ as a binary function, rather than implementing the average theory, as in [13]. The reason is that binary TLS functions reflect the phenomenon of stopand-go at intersections, which has a significant impact on emissions and energy consumption.

The split ratios at intersections $\beta_{i} \in[0,1]$ represent the percentage of vehicles coming from the upstream cells that want to go in $i$. We assume that they are known, constant over time, and subject to

$$
\forall \in \mathcal{R}_{\text {exit }}^{\text {in }} \cup \mathcal{R}_{\text {exit }}^{\text {inner }}, \sum_{j \in \mathcal{N}_{i}} \beta_{j}=1
$$

A standard 4-way intersection illustrating the TLS functions and the split ratios is given in Fig. 1. In this particular case, $\beta_{c}+\beta_{d}=1$ and $\alpha_{a}(k)+\alpha_{b}(k)=1$. Note that in the proposed approach, the split ratios applied at intersections

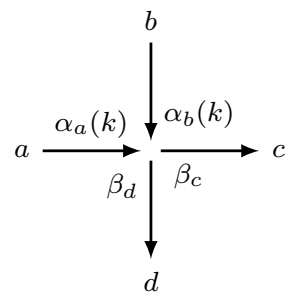

Fig. 1: Standard 4-ways intersection.

are the same for all the upstream roads, i.e. $\beta_{c}$ and $\beta_{d}$ are applied to the outflows of $a$ and $b$.

We define $u_{k}=\left[u_{i}(k)\right]_{i \in \mathcal{R}}$ as the vector containing all the cells' speed limits at time step $k$. Note that all the cells from the same road have the same speed limit. Similarly, the vectors $\rho_{k}=\left[\rho_{i}(k)\right]_{i \in \mathcal{R}}$ and $Q_{k}=\left[Q_{i}(k)\right]_{i \in \mathcal{R}_{\text {enter }}^{\text {in }}}$ contain respectively all cells' vehicle densities and the number of vehicles queuing in front of each network entering road at time step $k$. The system state is defined as

$$
x_{k}=\left[\rho_{k-1}, \rho_{k}, Q_{k}\right]
$$

It includes previous densities $\rho_{k-1}$, as they are necessary to estimate vehicles' acceleration (cf. Section II-B). We define the demand $D_{i}$ and supply $S_{i}$ of each cell $i$ as

$$
\begin{aligned}
D_{i}(k) & =\min \left\{u_{i}(k) \rho_{i}(k), \varphi^{\mathrm{M}}\left(u_{i}(k)\right)\right\} \\
S_{i}(k) & =\min \left\{\varphi^{\mathrm{M}}\left(u_{i}(k)\right), w\left(\rho^{\mathrm{M}}-\rho_{i}(k)\right)\right\}
\end{aligned}
$$

Similarly, we define $d_{i}(k)$ and $s_{i}(k)$ as the demand of the cells upstream the network and the supply of the cells downstream the network, respectively.

Based on the triangular FD introduced in [14], and represented in Fig. 2, the flow of vehicles $\varphi_{i}^{\text {out }}(k)$ exiting cell $i$ at time step $k$ is calculated as indicated in Table II.

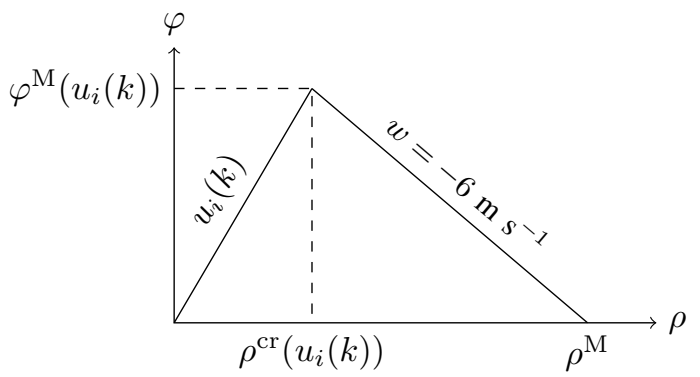

Fig. 2: FD of cell $i$ at time step $k$.

TABLE II: Cells outflows.

\begin{tabular}{cc}
\hline Set of cell & $\varphi_{i}^{\text {out }}(k)$ \\
\hline $\mathcal{R}_{\text {exit }}^{\text {out }}$ & $\min \left\{D_{i}(k), s_{i}(k)\right\}$ \\
$\mathcal{R}_{\text {exit }}^{\text {in }} \cup \mathcal{R}_{\text {exit }}^{\text {inner }}$ & $\alpha_{i}(k) \min \left\{D_{i}(k),\left\{\frac{S_{j}(k)}{\beta_{j}}\right\}_{j \in \mathcal{N}_{i}}\right\}$ \\
else & $\min \left\{D_{i}(k), S_{\mathcal{N}_{i}}(k)\right\}$ \\
\hline
\end{tabular}

As in (5b), $S_{\mathcal{N}_{i}}(k)$ is the supply of the cell indexed $\mathcal{N}_{i}$. 
Similarly, the vehicle flow entering cell $i$ at time step $k$, denoted $\varphi_{i}^{\text {in }}(k)$, is calculated as indicated in Table III.

TABLE III: Cells inflows.

\begin{tabular}{cc}
\hline Set of cell & $\varphi_{i}^{\text {in }}(k)$ \\
\hline $\mathcal{R}_{\text {enter }}^{\text {in }}$ & $\min \left\{d_{i}(k)+\frac{Q_{i}(k)}{\delta_{t}}, S_{i}(k)\right\}$ \\
$\mathcal{R}_{\text {enter }}^{\text {inner }} \cup \mathcal{R}_{\text {enter }}^{\text {out }}$ & $\beta_{i} \sum_{r \in \mathcal{P}_{i}} \varphi_{r}^{\text {out }}(k)$ \\
else & $\varphi_{\mathcal{P}_{i}}^{\text {out }}(k)$ \\
\hline
\end{tabular}

The dynamics of the system is described by the state vector field $f$ defined as follows

$$
x_{k+1}=f\left(x_{k}, u_{k}\right)
$$

where

$$
f: x_{k}, u_{k} \rightarrow\left(\begin{array}{c}
{\left[\rho_{i}(k)\right]_{i \in \mathcal{R}}} \\
{\left[\rho_{i}(k)+\frac{\delta_{t}}{\delta_{x}}\left(\varphi_{i}^{\text {in }}(k)-\varphi_{i}^{\text {out }}(k)\right)\right]_{i \in \mathcal{R}}} \\
{\left[Q_{i}(k)+\delta_{t}\left(d_{i}(k)-\varphi_{i}^{\text {in }}(k)\right)\right]_{i \in \mathcal{R}_{\text {enter }}^{\text {in }}}}
\end{array}\right)
$$

In order to guarantee the descriptive ability of the spatiotemporally discrete model, the Courant-Friedrichs-Lewy (CFL) condition [15] needs to be satisfied. It is expressed as

$$
2 \delta_{t} u_{\max } \leq \delta_{x}
$$

\section{B. Method to estimate the vehicles' acceleration}

Road traffic emissions and energy consumption can be estimated from vehicles kinematics (speed and acceleration). Based on the FD hypothesis, the traffic speed in cell $i$ can be expressed as

$$
v_{i}(k)=\min \left\{u_{i}(k), w \frac{\rho^{\mathrm{M}}-\rho_{i}(k)}{\rho_{i}(k)}\right\}
$$

The acceleration is not directly provided by traffic flow models. Thus, we propose to estimate it following the approach presented in [11]. This method considers that each vehicle is subject to either a temporal or a spatial acceleration.

The temporal acceleration is experienced by the vehicles that stay within the same cell from one time step to the next one. It reflects the speed variation within a cell. For each cell $i$, the acceleration $a_{i}^{\text {temp }}$ and the number of vehicles $N_{i}^{\text {temp }}$ subject to this acceleration are

$$
\begin{aligned}
a_{i}^{\text {temp }}(k) & =\frac{v_{i}(k)-v_{i}(k-1)}{\delta_{t}} \\
N_{i}^{\text {temp }}(k) & =\delta_{x} \rho_{i}(k)-\delta_{t} \varphi_{i}^{\text {out }}(k)
\end{aligned}
$$

The spatial acceleration is experienced by the vehicles that move from one cell to another one. It reflects the speed difference between adjacent cells. For each cell $i$, the acceleration $a_{r, i}^{\text {spat }}$ from its upstream cell $r$ and the number of vehicles $N_{r, i}^{\text {spat }}$ subject to this acceleration are

$$
\begin{aligned}
a_{r, i}^{\text {spat }}(k) & =\frac{v_{i}(k)-v_{r}(k-1)}{\delta_{t}} \\
N_{r, i}^{\text {spat }}(k) & = \begin{cases}\beta_{i} \delta_{t} \varphi_{r}^{\text {out }}(k) & \text { if } i \in \mathcal{R}_{\text {enter }}^{\text {inner }} \cup \mathcal{R}_{\text {enter }}^{\text {out }} \\
\delta_{t} \varphi_{i}^{\text {in }}(k) & \text { if } i \in \mathcal{R}_{\text {enter }}^{\text {in }} \\
\delta_{t} \varphi_{r}^{\text {out }}(k) & \text { else }\end{cases}
\end{aligned}
$$

We introduce acceleration lower and upper bounds, respectively denoted $a_{\min }$ and $a_{\max }$, to be realistic and remain in the domain of definition of the energetic model.

\section{SPEED LIMITS CONTROL STRATEGY}

In this section, we present an NMPC strategy aimed at reducing the environmental impact and improving the traffic performance, through VSL.

\section{A. Model Predictive Control}

Model predictive control (MPC) is a dynamic real-time control strategy that finds system inputs, every $\Delta_{c}$, optimizing an objective function calculated along a prediction horizon $\Delta_{p}=\kappa \Delta_{c}$.

At each control time step $k$, the controller receives the measured state of the system $x_{k}$ and determines an optimal control $u$ for the upcoming horizon $\Delta_{p}$. In our case, the MPC is parameterized as follows

- speed limits are constant between two successive control time steps;

- all cells from the same road have the same speed limit;

- to reduce the degree of freedom of the system, we propose to regulate the speed limits of several roads with the same variable, instead of having one variable per road. Hence, we define $n_{\mathrm{MPC}}<n$ sets of roads $\mathcal{R}^{1}, \ldots, \mathcal{R}^{n_{\mathrm{MPC}}}$, each set being constituted of roads subject to the same speed limits. A counterpart of this point is that the solution obtained may be suboptimal.

In other words, the controller solves the following optimization problem at each control time step $k$

$$
\min _{u_{k}, u_{k+\Delta_{c}}, \ldots, u_{k+(\kappa-1) \Delta_{c}}} \sum_{h=0}^{\Delta_{p}-1} J\left(x_{k+h}, u_{k+h}\right)
$$

where $J$ is the objective function defined in Section III$\mathrm{B}$, and the system state $x_{k+h}$ is obtained from $x_{k}$ using the state vector field $f$. A representation of this control approach is given in Fig. 3.

The control horizon is set to $\Delta_{c}=5 \mathrm{~min}$, so as to have a compromise between the flexibility of the controller and the comfort of road users. In order to ensure the robustness of the controller, we propose to consider $\kappa=6$ (i.e. $\Delta_{p}=30 \mathrm{~min}$ ). The speed limits lower and upper bounds are respectively set to $u_{\min }=20 \mathrm{~km} / \mathrm{h}$ and $u_{\max }=50 \mathrm{~km} / \mathrm{h}$. The controller uses the limited-memory Broyden-Fletcher-Goldfarb-Shanno (BFGS) minimization algorithm. 

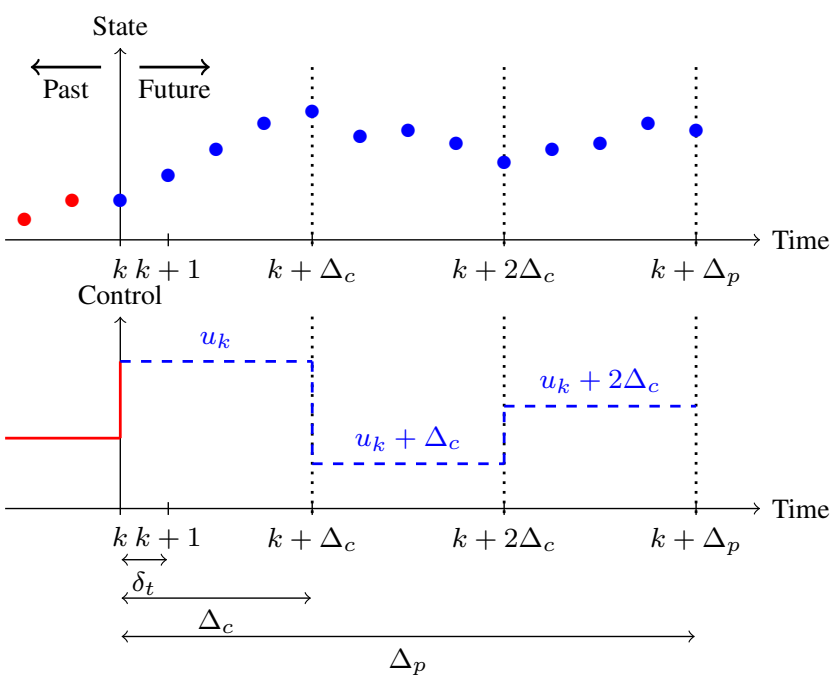

Fig. 3: Representation of the NMPC framework, for $\kappa=3$.

\section{B. Objective function}

Several objective functions can be considered for traffic eco-management [1]. Usually, these approaches consider weighted sums of ecological and traffic efficiency metrics, that can be antagonistic. The purpose is to avoid trivial solutions, and find a compromise between energy efficiency and traffic performance. In this study, we consider

$$
J\left(x_{k}, u_{k}\right)=\lambda \frac{E\left(x_{k}, u_{k}\right)}{E_{\text {norm }}}-(1-\lambda) \frac{T T D\left(x_{k}, u_{k}\right)}{T T D_{\text {norm }}}
$$

where $E$ and $T T D$ are nonlinear functions calculating respectively the total fuel consumption in the network (in liters per second), and the distance traveled by all the vehicles in the network (in meters), over $\delta_{t} . E_{\text {norm }}$ and $T T D_{\text {norm }}$ are normalization coefficients, and $\lambda$ is a weighting coefficient.

$E$ and $T T D$ are defined as follows

$$
\begin{aligned}
E\left(x_{k}, u_{k}\right) & =\sum_{i \in \mathcal{R}} E_{i}\left(x_{k}, u_{k}\right) \\
T T D\left(x_{k}, u_{k}\right) & =\delta_{t} \delta_{x} \sum_{i \in \mathcal{R}} \rho_{i}(k) v_{i}(k)
\end{aligned}
$$

$E_{i}\left(x_{k}, u_{k}\right)$ denotes the fuel consumption, in liters per second, in cell $i$ during time interval $\left[k \delta_{t},(k+1) \delta_{t}\right]$. It is calculated using the VT-macro approach [11], described as

$$
E_{i}\left(x_{k}, u_{k}\right)=E_{i}^{\mathrm{temp}}\left(x_{k}, u_{k}\right)+\sum_{r \in \mathcal{P}_{i}} E_{r, i}^{\mathrm{spat}}\left(x_{k}, u_{k}\right)
$$

where

$$
\begin{gathered}
E_{i}^{\text {temp }}\left(x_{k}, u_{k}\right)=N_{i}^{\text {temp }}(k) \xi\left(a_{i}^{\text {temp }}(k)\right) \cdot M_{\text {fuel }} \cdot \xi\left(v_{i}(k)\right)^{T} \\
E_{r, i}^{\text {spat }}\left(x_{k}, u_{k}\right)=N_{r, i}^{\text {spat }}(k) \xi\left(a_{r, i}^{\text {spat }}(k)\right) \cdot M_{\text {fuel }} \cdot \xi\left(v_{i}(k)\right)^{T}
\end{gathered}
$$

$M_{\text {fuel }}$ is the model regression parameters matrix [11], [16]. It can be calibrated for fuel consumption, but also for pollutant emissions (for example, $M_{N O x}$ for NOx emissions).
The function $\xi$ is defined as

$$
\xi(x)=\left[\begin{array}{llll}
1 & x & x^{2} & x^{3}
\end{array}\right]
$$

Note that $M_{\text {fuel }}$ can depend on the acceleration, in which case there is a set of parameters $M_{\text {fuel,+ }}$ calibrated for positive accelerations, and a set of parameters $M_{\text {fuel,-- calibrated }}$ for negative accelerations.

When $a_{i}^{\text {temp }}(k)$ or $a_{r, i}^{\text {temp }}(k)$ exceeds $a_{\max }$, (16) becomes

$$
\begin{aligned}
& E_{i}^{\text {temp }}(k)=N_{i}^{\text {temp }}(k) \frac{a_{i}^{\text {temp }}(k)}{a_{\max }} \xi\left(a_{\max }\right) M_{\text {fuel }} \xi\left(v_{i}(k)\right)^{T} \\
& E_{r, i}^{\text {spat }}(k)=N_{r, i}^{\text {spat }}(k) \frac{a_{r, i}^{\text {spat }}(k)}{a_{\max }} \xi\left(a_{\max }\right) M_{\text {fuel }} \xi\left(v_{i}(k)\right)^{T}
\end{aligned}
$$

\section{Simulation AND RESUlts}

In this section, we evaluate the control performance by comparing the following cases

- case ref: constant speed limit $u_{\text {ref }}$ (uncontrolled);

- case vsl: VSL managed by the controller (controlled).

\section{A. Traffic performance metrics}

In order to evaluate the impact of the VSL strategy on the environment, and on the traffic performance within and at the boundaries of the network, we propose to connect it to a performance evaluation model.

Additional metrics, which are not in the objective function, are introduced and listed in Table IV. Depending on the value of the weighting coefficient $\lambda$, a trade-off is expected between the ecological metrics ( $E$ and $N O x$ ), and the traffic performance metrics. We propose to set $\lambda=0.5$.

Simulations are run considering the baseline (ref) and the controlled ( $v s l)$ scenarios. Based on the Symmetric Mean Absolute Percentage Error (SMAPE) approach, we define the relative improvement of each metric $X$, denoted $\eta_{X}$ as

- $\eta_{X}=-\frac{X_{\mathrm{ref}}-X_{\mathrm{vsl}}}{\left(X_{\mathrm{ref}}+X_{\mathrm{vsl}}\right) / 2}$, if $X$ is to be maximized;

- $\eta_{X}=\frac{X_{\text {ref }}-X_{\mathrm{vsl}}}{\left(X_{\text {ref }}+X_{\text {refvis }}\right) / 2}$, if $X$ is to be minimized.

where $X_{\text {ref }}$ and $X_{\mathrm{vsl}}$ are the values of metric $X$ evaluated in cases ref and $v s l$, respectively. All metrics are to be minimized, except $T T D$ and $S o D$, which are to be maximized.

If $\eta_{X}>0$, the control is beneficial for metric $X$. If $\eta_{X}<0$, the control has degraded metric $X$.

\section{B. Simulation parameters}

Let us consider a grid network composed of $n=40$ identical one-way roads of $300 \mathrm{~m}$ each, as represented in Fig. 4. The network is composed of 8 entering roads and 8 exiting roads. The duration of simulation is set to $T=1 \mathrm{~h}$.

We consider an initial density of vehicles $\rho_{0}$, and a constant traffic demand on each entering road $d=1200 \mathrm{veh} / \mathrm{h}$, i.e. a global demand on the network of $9600 \mathrm{veh} / \mathrm{h}$.

In order to alter the symmetry of the network, we propose to introduce privileged directions: the split ratios $\beta_{i}$ are set to 0.7 for the vertical roads, and 0.3 for the horizontal roads. 
TABLE IV: Metrics.

\begin{tabular}{|c|c|c|c|}
\hline Symbol & Unit & Description & Formula \\
\hline$E$ & $\mathrm{~L}$ & Total fuel consumption. & $\delta_{t} \sum_{k=1}^{T} E(k)($ cf. $14-18)$ \\
\hline$N O x$ & $\mathrm{~kg}$ & Total NOx emissions. & $\delta_{t} \sum_{k=1}^{T} N O x(k)(\mathrm{cf} .14-18)$ \\
\hline \multirow{2}{*}{$E_{\text {veh }}$} & \multirow[t]{2}{*}{$\mathrm{L}$} & \multirow{2}{*}{ Average fuel consumption per vehicle that has been in the network. } & $E$ \\
\hline & & & $\delta_{x} \sum_{i \in \mathcal{R}} \rho_{i}(0)+\delta_{t} \sum_{k=1}^{T} \sum_{i \in \mathcal{R}_{\text {enter }}^{\text {in }}} \varphi_{i}^{\text {in }}(k)$ \\
\hline \multirow{2}{*}{$N O x_{\mathrm{veh}}$} & \multirow[t]{2}{*}{$\mathrm{kg}$} & \multirow[t]{2}{*}{ Average NOx emissions per vehicle that has been in the network. } & NOx \\
\hline & & & $\delta_{x} \sum_{i \in \mathcal{R}} \rho_{i}(0)+\delta_{t} \sum_{k=1}^{T} \sum_{i \in \mathcal{R}_{\text {enter }}^{\text {in }}} \varphi_{i}^{\text {in }}(k)$ \\
\hline$T T D$ & $\mathrm{~m}$ & Distance traveled by all vehicles in the network. & $\sum_{k=1}^{T} T T D(k)(\mathrm{cf} .14 \mathrm{~b})$ \\
\hline$T T S_{\text {net }}$ & $\mathrm{s}$ & Total time spent by all vehicles in the network. & $\delta_{t} \delta_{x} \sum_{k=1}^{T} \sum_{i \in \mathcal{R}} \rho_{i}(k)$ \\
\hline$T T S_{\text {queue }}$ & $\mathrm{s}$ & $\begin{array}{l}\text { Total time spent by vehicles queuing in front of the entering roads of the network } \\
\text { (outside the network). }\end{array}$ & $\delta_{t} \sum_{k=1}^{T} \sum_{i \in \mathcal{R}^{i n}} Q_{i}(k)$ \\
\hline$Q o S$ & - & Total number of vehicles queuing outside the network at the end of the simulation. & $\sum_{i \in \mathcal{R}_{\text {enter }}^{\text {in }}} Q_{i}(T)$ \\
\hline \multirow{2}{*}{ SoD } & \multirow{2}{*}{-} & \multirow{2}{*}{$\begin{array}{l}\text { Ratio between the number of vehicles that have entered the network and those aiming } \\
\text { at entering the network. }\end{array}$} & $\delta_{t} \sum_{k=1}^{T} \sum_{i \in \mathcal{R}_{\text {enter }}^{\text {in }}} \varphi_{i}^{\text {in }}(k)$ \\
\hline & & & $\overline{Q o S}+\delta_{t} \sum_{k=1}^{T} \sum_{i \in \mathcal{R}_{\text {enter }}^{\text {in }}} \varphi_{i}^{\text {in }}(k)$ \\
\hline
\end{tabular}

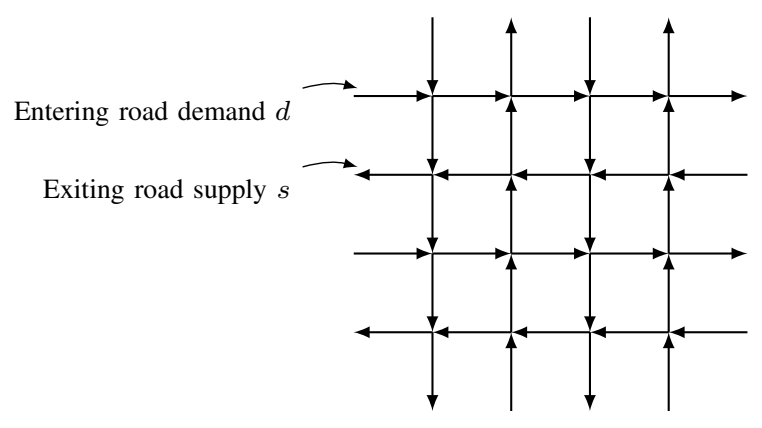

Fig. 4: Synthetic urban road network.

The traffic light signals at each intersection have cycles of $1 \mathrm{~min}$, without any offset, characterized as follows

- $30 \mathrm{~s}$ of green for the horizontal roads;

- $30 \mathrm{~s}$ of green for the vertical roads;

In order to find a compromise between the computational burden and the traffic model precision, we propose to consider a discretization of $n_{\text {cells }}=5$ cells per road.

\section{Results}

For environmental reasons, more and more cities have made the choice to generalize the $30 \mathrm{~km} / \mathrm{h}$ speed limit [17]. Hence, we propose to calculate the metrics improvements with respect to both $u_{\text {ref }}=50 \mathrm{~km} / \mathrm{h}$ and $u_{\text {ref }}=30 \mathrm{~km} / \mathrm{h}$. In each case, simulations are run for an initial density $\rho_{0}$ varying from 0 to $0.8 \rho^{\mathrm{M}}$ in order to get a broad view of the control performance. The results are given in Fig. 5.

For initial densities $\rho_{0} \leq 0.7 \rho^{\mathrm{M}}$, when compared to the uncontrolled case with $u_{\text {ref }}=50 \mathrm{~km} / \mathrm{h}$ (cf. Fig. 5a), the controller improves the ecological metrics $\left(\eta_{E_{\mathrm{veh}}}\right.$ and $\eta_{N O x_{\mathrm{veh}}}$ are positive), without deteriorating the traffic performance within and at the boundaries of the network. When compared to the case with $u_{\text {ref }}=30 \mathrm{~km} / \mathrm{h}$ (cf. Fig. $5 \mathrm{~b}$ ), the solutions proposed by the controller constitute a compromise between the ecological metrics $\left(\eta_{E_{\mathrm{veh}}}\right.$ and $\eta_{N O x_{\mathrm{veh}}}$ are negative) and the traffic performance metrics, which are improved both within and at the boundaries of the network.

For initial densities $\rho_{0}>0.7 \rho^{\mathrm{M}}$, all metrics start to improve significantly, with respect to both $u_{\text {ref }}=50 \mathrm{~km} / \mathrm{h}$ and $u_{\text {ref }}=30 \mathrm{~km} / \mathrm{h}$. The reason is that the NMPC prediction makes the VSL strategy able to avoid gridlocks, while both uncontrolled cases fail to prevent it.

An illustration of this is given in Fig. 6, where after $T=1 \mathrm{~h}$ of simulation, the uncontrolled case with $u_{\text {ref }}=50 \mathrm{~km} / \mathrm{h}$ gives rise to a gridlock, while the VSL control is able to maintain traffic fluidity.

Generally, it appears that the controller is more effective in congested situations, which is consistent with VSL approaches for freeways [6].

\section{CONCLUSIONS AND FUTURE WORKS}

In this work, an NMPC approach has been employed for ecological VSL in an urban network. For the chosen set of parameters, simulation results reveal that the controller improves ecological metrics when compared to the uncontrolled $50 \mathrm{~km} / \mathrm{h}$ speed limits scenario, and improves traffic performance metrics when compared to the uncontrolled $30 \mathrm{~km} / \mathrm{h}$ speed limits scenario. The controller is more efficient in congested situations, in particular via gridlock avoidance. This is consistent with VSL designs applied to highways, which are mostly effective in congested systems.

To go further, a validation using a microscopic traffic simulator should be performed to assess the accuracy of the controller, as well as the traffic and energetic models, 


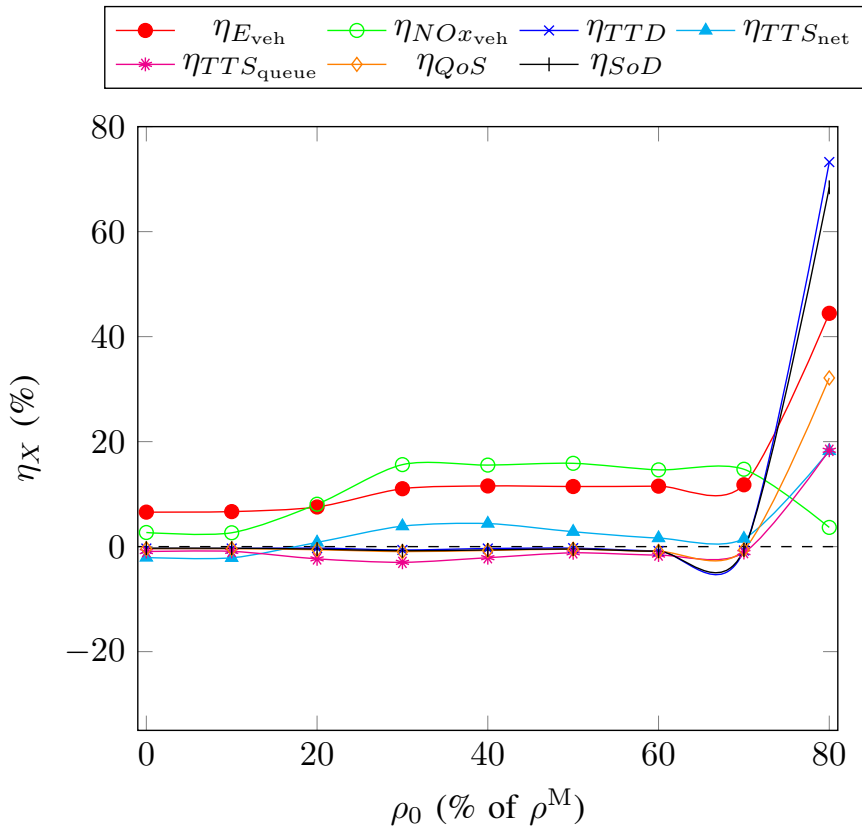

(a) $u_{\mathrm{ref}}=50 \mathrm{~km} / \mathrm{h}$

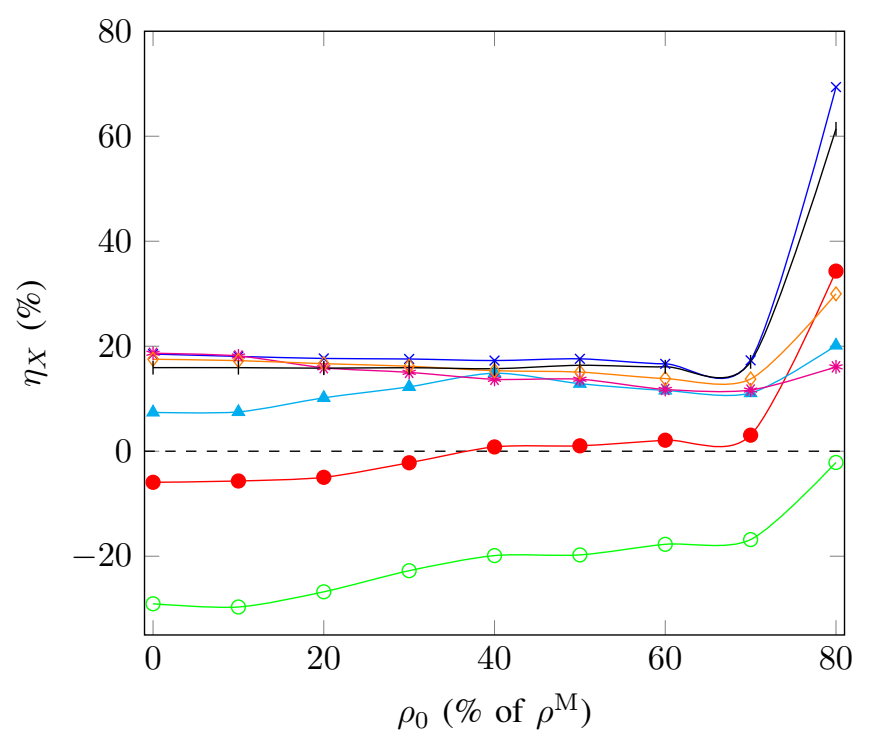

(b) $u_{\mathrm{ref}}=30 \mathrm{~km} / \mathrm{h}$

Fig. 5: $\eta_{X}$ as a function of $\rho_{0}$, for $d=1200 \mathrm{veh} / \mathrm{h}$.

the latter being very dependent on vehicles' accelerations. Future developments will coordinate VSL with TLS control to improve the sustainability and the performance of urban traffic. The controller should be robust and improve the situation both during congested and uncongested periods, while also being suitable for online implementation.

\section{REFERENCES}

[1] B. Othman, G. De Nunzio, D. Di Domenico, and C. Canudas-deWit, "Ecological traffic management: A review of the modeling and control strategies for improving environmental sustainability of road transportation," Annual Reviews in Control, 2019.

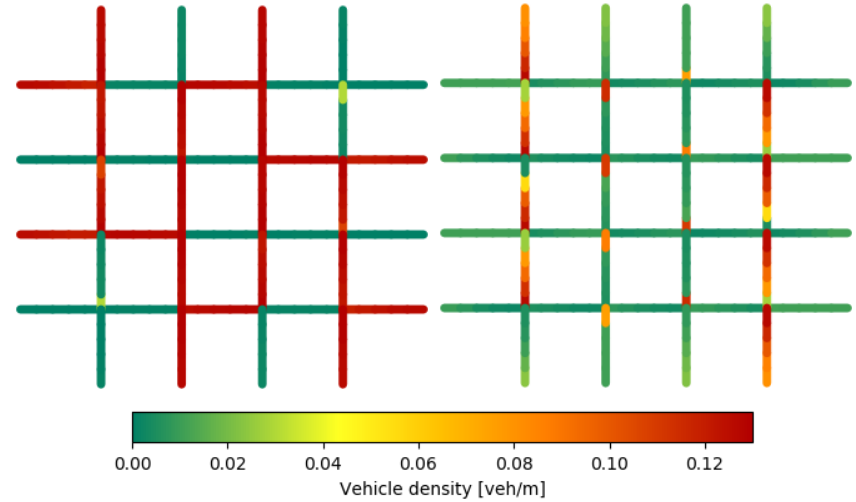

Fig. 6: Density distribution in the network after one hour of simulation, for $\rho_{0}=0.8 \rho^{\mathrm{M}}$, in the uncontrolled $u_{\text {ref }}=$ $50 \mathrm{~km} / \mathrm{h}$ (left) and controlled (right) scenarios.

[2] M. A. Taylor, "Network modelling of the traffic, environmental and energy effects of lower urban speed limits," Road \& Transport Research, vol. 9, no. 4, p. 48, 2000.

[3] M. Tajali and A. Hajbabaie, "Dynamic speed harmonization in urban street networks," Computer-Aided Civil and Infrastructure Engineering, 2018.

[4] G. De Nunzio, C. Canudas-deWit, and P. Moulin, "Urban traffic eco-driving: A macroscopic steady-state analysis," in 2014 European Control Conference (ECC), pp. 2581-2587, IEEE, 2014.

[5] E. Walraven, M. T. Spaan, and B. Bakker, "Traffic flow optimization: A reinforcement learning approach," Engineering Applications of Artificial Intelligence, vol. 52, pp. 203 - 212, 2016.

[6] M. Hadiuzzaman and T. Z. Qiu, "Cell transmission model based variable speed limit control for freeways," Canadian Journal of Civil Engineering, vol. 40, no. 1, pp. 46-56, 2013.

[7] S. K. Zegeye, B. De Schutter, J. Hellendoorn, E. A. Breunesse, and A. Hegyi, "A predictive traffic controller for sustainable mobility using parameterized control policies," IEEE Transactions on Intelligent Transportation Systems, vol. 13, no. 3, pp. 1420-1429, 2012.

[8] B. Khondaker and L. Kattan, "Variable speed limit: an overview," Transportation Letters, vol. 7, no. 5, pp. 264-278, 2015.

[9] M. Garavello, K. Han, and B. Piccoli, Models for vehicular traffic on networks, vol. 9. American Institute of Mathematical Sciences (AIMS), Springfield, MO, 2016.

[10] C. F. Daganzo, "The cell transmission model: A dynamic representation of highway traffic consistent with the hydrodynamic theory," Transportation Research Part B: Methodological, vol. 28, no. 4, pp. 269-287, 1994.

[11] S. Zegeye, B. De Schutter, J. Hellendoorn, E. Breunesse, and A. Hegyi, "Integrated macroscopic traffic flow, emission, and fuel consumption model for control purposes," Transportation Research Part C: Emerging Technologies, vol. 31, pp. 158-171, 2013.

[12] S. Godunov, "A difference scheme for numerical computation of discontinuous solution of hyperbolic equation," Math. Sbornik, vol. 47, pp. 271-306, 1959.

[13] P. Grandinetti, C. Canudas-de-Wit, and F. Garin, "An efficient onestep-ahead optimal control for urban signalized traffic networks based on an averaged cell-transmission model," in 2015 European Control Conference (ECC), pp. 3478-3483, IEEE, 2015.

[14] G. F. Newell, "A simplified theory of kinematic waves in highway traffic, part i: General theory," Transportation Research Part B: Methodological, vol. 27, no. 4, pp. 281-287, 1993.

[15] R. Courant, K. Friedrichs, and H. Lewy, "On the partial difference equations of mathematical physics," IBM journal of Research and Development, vol. 11, no. 2, pp. 215-234, 1967.

[16] M. Alsabaan, A. Nayak, K. Naik, and T. Khalifa, Applying Vehicular Networks for Reduced Vehicle Fuel Consumption and CO2 Emissions. INTECH Open Access Publisher, 2012.

[17] J. Bordarie, "Public policy of urban mobility: Impact of the history and practices on young drivers' social representation of $30 \mathrm{~km} / \mathrm{hr}$," Journal of Nonprofit \& Public Sector Marketing, vol. 29, no. 2, pp. 211-234, 2017. 